\title{
Autosomal recessive ataxia, Beauce type
}

INSERM

\section{Source}

INSERM. (1999). Orphanet: an online rare disease and orphan drug data base. Autosomal recessive ataxia, Beauce type. ORPHA:88644

Autosomal recessive ataxia, Beauce type is characterised by a slowly progressive pure cerebellar ataxia associated with dysarthria. It has been described in 53 individuals from 26 families of Canadian origin. The mode of transmission is autosomal recessive.

Positional cloning has led to the identification of several SYNE1 gene mutations. 